\title{
Short communication: Conversion of lactose and whey into lactic acid by engineered yeast
}

\author{
Timothy L. Turner, ${ }^{\star} \dagger$ Eunbee Kim,‡ ChangHoon Hwang,§ Guo-Chang Zhang, ${ }^{*} \dagger$ Jing-Jing Liu, ${ }^{\star} \dagger$ \\ and Yong-Su Jin* ${ }^{1}$ \\ *Department of Food Science and Human Nutrition, and \\ †Carl R. Woese Institute for Genomic Biology, and \\ ‡Department of Biochemistry, University of Illinois at Urbana-Champaign, Urbana 61801 \\ §Department of Food Science Technology, Chungnam National University, Daejeon 34134, South Korea
}

\begin{abstract}
Lactose is often considered an unwanted and wasted byproduct, particularly lactose trapped in acid whey from yogurt production. But using specialized microbial fermentation, the surplus wasted acid whey could be converted into value-added chemicals. The baker's yeast Saccharomyces cerevisiae, which is commonly used for industrial fermentation, cannot natively ferment lactose. The present study describes how an engineered $S$. cerevisiae yeast was constructed to produce lactic acid from purified lactose, whey, or dairy milk. Lactic acid is an excellent proof-of-concept chemical to produce from lactose, because lactic acid has many food, pharmaceutical, and industrial uses, and over 250,000 t are produced for industrial use annually. To ferment the milk sugar lactose, a cellodextrin transporter $(C D T-1$, which also transports lactose) and a $\beta$-glucosidase (GH1-1, which also acts as a $\beta$-galactosidase) from Neurospora crassa were expressed in a $S$. cerevisiae strain. A heterologous lactate dehydrogenase (encoded by $l d h A$ ) from the fungus Rhizopus oryzae was integrated into the $C D T-1 / G H 1-1$-expressing strain of $S$. cerevisiae. As a result, the engineered strain was able to produce lactic acid from purified lactose, whey, and store-bought milk. A lactic acid yield of $0.358 \mathrm{~g} / \mathrm{g}$ of lactose was achieved from whey fermentation, providing an initial proof of concept for the production of value-added chemicals from excess industrial whey using engineered yeast.
\end{abstract}

Key words: lactic acid, yeast, whey, lactose

\section{Short Communication}

Whey is a significant and troublesome byproduct of the dairy industry (Siso, 1996). In particular, cheese

Received July 26, 2016.

Accepted September 9, 2016.

${ }^{1}$ Corresponding author: ysjin@illinois.edu production can generate up to $9 \mathrm{~kg}$ of whey per $1 \mathrm{~kg}$ of cheese, and the disaccharide lactose serves as the major constituent of the permeate after whey filtration (González-Siso et al., 2015). Acid whey is particularly problematic, because it is produced in excess as a byproduct of Greek yogurt production and is difficult for the yogurt industry to efficiently reuse or discard, in part due to the relatively low $\mathrm{pH}$ of acid whey $(\mathrm{pH}$ <5.1) (Miller et al., 2006; Elliott, 2013). In response, one creative solution for the problem of whey as a dairy industry byproduct is to use engineered microbes to ferment the lactose in the whey to produce valueadded fuels and chemicals (González-Siso et al., 2015). However, even this solution is not easy, because it is recommended that the whey concentration used should contain up to $120 \mathrm{~g} / \mathrm{L}$ lactose to minimize downstream purification costs (Siso, 1996; Arif et al., 2008). Therefore, an engineered microbe must not only be able to withstand the acidity of acid whey and osmotic stress induced by a high concentration of lactose, it must also produce a target product at a high yield and productivity.

Recently, an engineered strain of Saccharomyces cerevisiae baker's yeast was developed that can efficiently produce ethanol at a yield of $0.361 \mathrm{~g} / \mathrm{g}$ of lactose from whey medium containing $150 \mathrm{~g} / \mathrm{L}$ of lactose under anaerobic conditions (Liu et al., 2016). Most yeast, including $S$. cerevisiae, cannot natively ferment lactose. This lactose-fermenting strain was created by introducing and expressing 2 heterologous genes cloned from the fungus Neurospora crassa: a lactose transporter $(C D T-1)$ to transport lactose into the yeast cell and a $\beta$-galactosidase (GH1-1) to cleave the lactose disaccharide into glucose and galactose (Galazka et al., 2010; Oh et al., 2016).

Here, we expand on the initial concept of producing an endogenous product of $S$. cerevisiae (ethanol) toward producing a heterologous value-added product (lactic acid). This study provides proof of concept for the conversion of purified lactose, milk, and whey into lactic 
acid using an engineered S. cerevisiae strain. Yeasts, especially S. cerevisiae, are ideal microbes for industrial chemical production because of their tolerance of high external osmotic stress (such as high sugar concentrations) and low $\mathrm{pH}$ (essential for overproducing organic acids); because they are generally recognized as safe; and because a wide range of genetic perturbation tools are available to study, introduce, and delete genes in the yeast genome (Laluce et al., 2012). Furthermore, the theoretical maximum yield of lactic acid from lactose is $1.00 \mathrm{~g} / \mathrm{g}$ of lactose due to the lack of carbon loss throughout the metabolic pathway. Perhaps most importantly, oxygen is theoretically unnecessary for this process to occur, so large-scale fermentation of lactose to lactic acid could be conducted entirely anaerobically, significantly reducing the costs accrued if oxygen were added (Porro et al., 1995).

We expressed a heterologous lactate dehydrogenase gene (ldhA), cloned from the fungus Rhizopus oryzae, into the $c d t-1-$ and gh1-1-expressing $S$. cerevisiae as previously reported (Turner et al., 2016). Using a fungal-sourced $l d h A$ instead of a bacterial lactate dehydrogenase in yeast was advantageous, because most bacterial lactate dehydrogenase enzymes perform optimally at an internal cellular $\mathrm{pH}$ range of $\sim 5$, but eukaryotic lactate dehydrogenase performs optimally in the neutral pH range (Skory, 2003). Because S. cerevisiae intracellular $\mathrm{pH}$ is maintained near neutral even in the presence of a low extracellular $\mathrm{pH}$ (Breeuwer and Abee, 2000), the fungal $R$. oryzae ldhA is preferential for integration in the $c d t-1 / g h 1-1$-expressing strain. The $l d h A$ was integrated into the $S$. cerevisiae chromosome using the pITy3-ldhA-G418 plasmid to integrate at Ty $\delta$ loci (Parekh et al., 1996) and selected using G418 (geneticin) as an antibiotic selection pressure. For all experiments, 2 engineered $S$. cerevisiae yeast strains were used, both expressing the $c d t-1$ and gh1-1 lactosefermenting pathway: EJ4 (no $l d h A$; Wei et al., 2015) and EJ4L (expressing ldhA; Turner et al., 2016). The EJ4 strain served as the control, which in all fermentations was unable to produce lactic acid. Importantly, the native ethanol production pathway consisting of pyruvate decarboxylase and alcohol dehydrogenase was not disrupted in either the EJ4 or EJ4L strains, allowing the yeast cells to produce ethanol as necessary (Turner et al., 2016).

Stock cultures of $S$. cerevisiae were maintained on yeast extract-peptone dextrose (YPD) agar plates $(2 \%$ agar, $1 \%$ yeast extract, $2 \%$ peptone, and $2 \%$ glucose) at $4^{\circ} \mathrm{C}$. Yeast precultures were grown in YPD40 medium at $300 \mathrm{rpm}$ and harvested at mid-log phase and then washed twice with sterilized water to prepare inocula for fermentation. All flask fermentations were conducted using $25 \mathrm{~mL}$ of medium in a $125-\mathrm{mL}$ Erlenmeyer flask maintained at $30^{\circ} \mathrm{C}$ on a 100-rpm MaxQ4000 orbital shaker (Thermo Fisher Scientific Inc., Waltham, MA). Flask fermentations in Figure 1 were performed using yeast-peptone medium containing $40 \mathrm{~g} / \mathrm{L}$ of purified lactose (Sigma-Aldrich, St. Louis, MO) with an initial cell concentration of $\sim 1$ ( 0.47 dry cell weight/ $\mathrm{mL}$, optical density at $600 \mathrm{~nm}$ ). The YP medium and lactose were sterilized separately via autoclave. Flask fermentations in Figure 2 were performed using Horizon Organic brand, shelf-stable, $1 \%$ fat, organic cow milk (Whitewave Services Inc., Denver, CO; lot 36-4016 03:37), which was used before the printed "best before" date. Because the milk was shelf-stable, we did not autoclave it before use. Flask fermentations in Figure 3 contained YP medium with $\sim 80 \mathrm{~g} / \mathrm{L}$ whey from bovine milk (Sigma-Aldrich), which resulted in $\sim 50 \mathrm{~g} / \mathrm{L}$ of free lactose. Due to suspected microbial contamination when using non-autoclaved whey (data not shown), all results in Figure 3 are from autoclaved whey medium. Approximately $35 \mathrm{~g} / \mathrm{L}$ of calcium carbonate $\left(\mathrm{CaCO}_{3}\right)$ was used as a neutralizing agent for experiments in Figure 1 and Figure 3. All experiments were repeated independently in duplicate, with variations indicated with error bars.

Yeast cell concentration was monitored by optical density at $600 \mathrm{~nm}$ using a Biomate 3 UV-visible spectrophotometer (Thermo Fisher Scientific Inc.). Lactose, glycerol, acetate, ethanol, and lactic acid concentrations

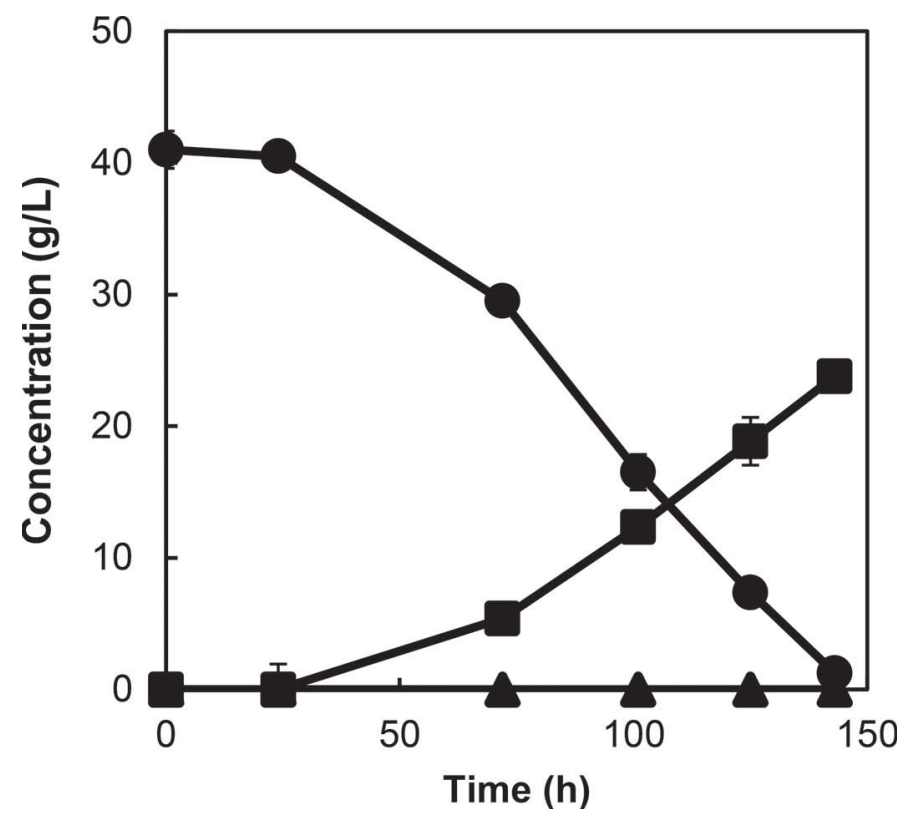

Figure 1. Fermentation profile of the EJ4L strain grown in yeast peptone medium containing $40 \mathrm{~g} / \mathrm{L}$ of lactose as the sole carbon source and $35 \mathrm{~g} / \mathrm{L}$ of $\mathrm{CaCO}_{3}$, as shown by concentrations of lactose $(\mathbf{)})$, lactic acid $(\boldsymbol{\square})$, and ethanol $(\boldsymbol{\Delta})$. Results are the averages of duplicate experiments; error bars indicate standard deviations. 


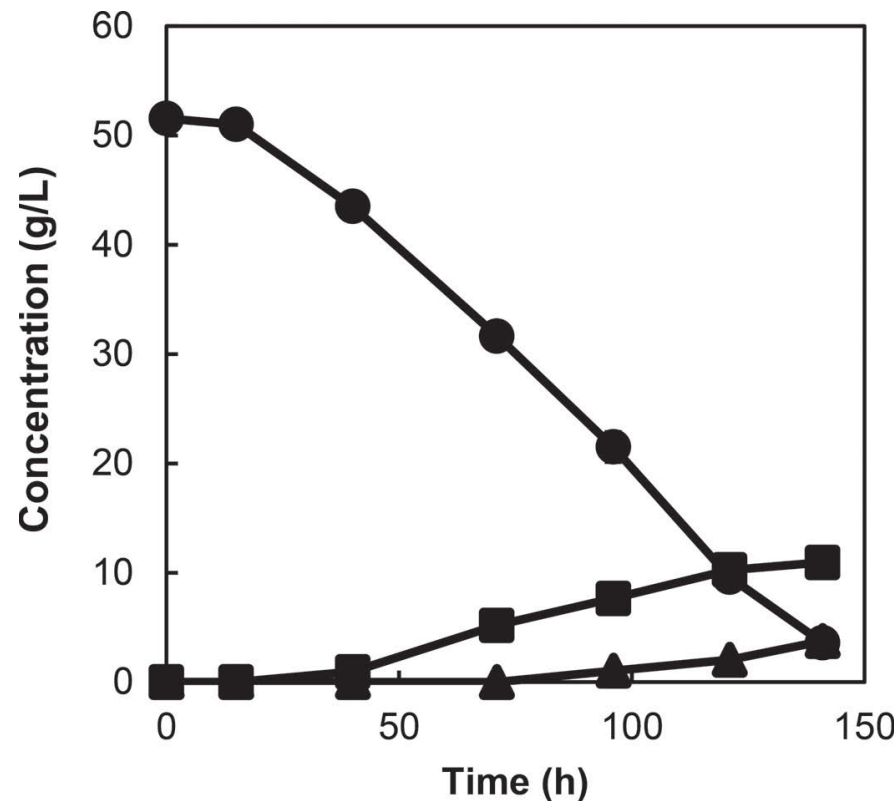

Figure 2. Fermentation profile of the EJ4L strain grown in shelfstable cow milk with no additional supplements, as shown by concentrations of lactose $(\boldsymbol{\bullet})$, lactic acid $(\mathbf{\square})$, and ethanol $(\mathbf{\Lambda})$. Results are the averages of duplicate experiments; error bars indicate standard deviations.

were determined using a 1200 Infinity Series HPLC instrument (Agilent Technologies Inc., Santa Clara, CA) equipped with a refractive index detector using a Rezex ROA-Organic Acid $\mathrm{H}^{+}(8 \%)$ column (Phenomenex Inc., Torrance, CA). The column was eluted with $0.005 \mathrm{~N}$ $\mathrm{H}_{2} \mathrm{SO}_{4}$ at a flow rate of $0.6 \mathrm{~mL} / \mathrm{min}$ at $50^{\circ} \mathrm{C}$. The $\mathrm{pH}$ value was monitored using a PHM210 $\mathrm{pH}$ meter (Ra-

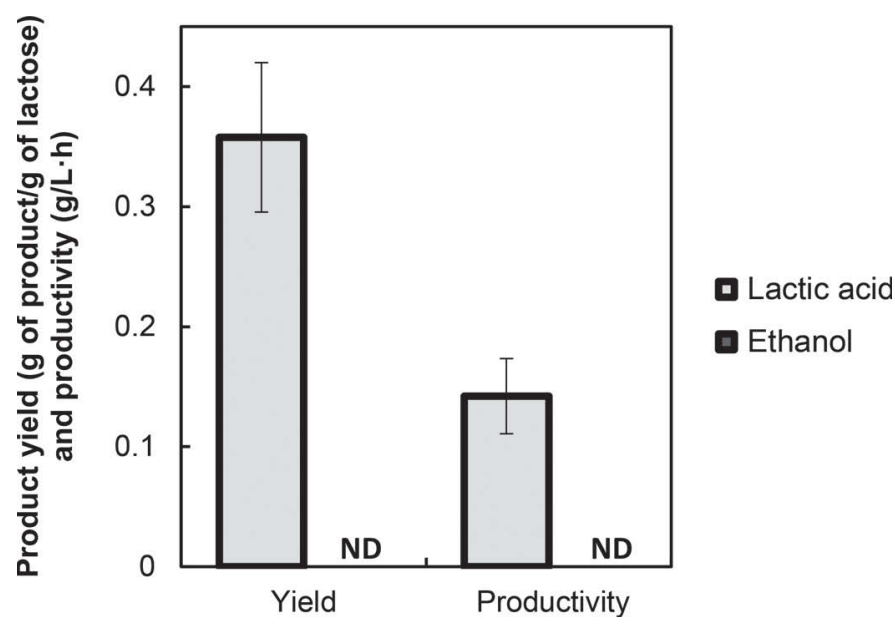

Figure 3. Lactic acid and ethanol yields (g of product/g of lactose) and productivities $(\mathrm{g} / \mathrm{L} \cdot \mathrm{h})$ of the EJ4L strain grown for $100 \mathrm{~h}$ in yeast peptone medium containing $\sim 80 \mathrm{~g} / \mathrm{L}$ of whey and $35 \mathrm{~g} / \mathrm{L}$ of $\mathrm{CaCO}_{3}$. $\mathrm{ND}=$ no detection of ethanol. Results are the averages of duplicate experiments; error bars indicate standard deviations. diometer Analytical SAS, Lyon, France) with an attached Accumet 13-620-290 pH probe (Thermo Fisher Scientific Inc.).

We used purified lactose in our initial test of the ability of the engineered $S$. cerevisiae strain (EJ4L) to produce lactic acid from lactose. Previous results indicated that a CDT-1/GH1-1-expressing yeast strain without a functional lactate dehydrogenase gene could produce ethanol at a yield of $0.361 \mathrm{~g} / \mathrm{g}$ of lactose under anaerobic conditions (Liu et al., 2016). With the introduction of the $l d h A$ gene into our EJ4 yeast strain, the EJ4L strain could efficiently produce lactic acid from lactose. From $41 \mathrm{~g} / \mathrm{L}$ of lactose, the EJ4L strain could produce $23.77 \mathrm{~g} / \mathrm{L}$ of lactic acid in $143 \mathrm{~h}$, resulting in a yield of $0.58 \mathrm{~g}$ of lactic acid/g of lactose and a productivity of $0.17 \mathrm{~g} / \mathrm{L} \cdot \mathrm{h}$ (Figure 1 ). We detected no ethanol production during the fermentation. The $\mathrm{pH}$ of the medium remained above 5.0 throughout the fermentation (data not shown) despite significant lactic acid production, because of the addition of $35 \mathrm{~g} / \mathrm{L} \mathrm{CaCO}_{3}$, which acted as a neutralizing agent. This was the first record of direct conversion of lactose to lactic acid using an engineered $S$. cerevisiae strain.

After confirming the successful conversion of lactose to lactic acid, we assessed whether lactose in cow milk could be efficiently converted to lactic acid. One difficulty in the dairy industry is finding an efficient use for waste milk from sick cattle (Stabel, 2001). Obviously, milk from contaminated cows cannot be fed to humans or even other livestock without significant risk for spreading disease. However, using modern biotechnology, it may become possible to convert the lactose in milk from contaminated cows into value-added products, such as lactic acid. Therefore, we tested this concept using shelf-stable cow's milk without other supplements or modifications (such as the addition of YP medium or $\mathrm{CaCO}_{3}$ ) as a proof of concept (Figure 2 ). By $121 \mathrm{~h}$, from $\sim 52 \mathrm{~g} / \mathrm{L}$ of lactose present in the milk, the EJ4L strain had consumed $\sim 42 \mathrm{~g} / \mathrm{L}$, producing $\sim 10.3 \mathrm{~g} / \mathrm{L}$ of lactic acid and resulting in a yield of $\sim 24.4 \mathrm{~g}$ of lactic acid/g of lactose and a productivity of $0.085 \mathrm{~g} / \mathrm{L} \cdot \mathrm{h}$ (Figure 2). Through $71 \mathrm{~h}$, we detected no ethanol production, but by $140 \mathrm{~h}$, the rate of ethanol production had begun to exceed the rate of lactic acid production, suggesting that as the lactic acid concentration increased, the metabolic flux shifted away from lactic acid production and toward ethanol production. Because we used no buffer or neutralizing agent $\left(\mathrm{CaCO}_{3}\right)$ in the milk medium and because lactate dehydrogenase can undergo feedback inhibition as lactic acid accumulates and $\mathrm{pH}$ decreases, the late ethanol production may have been due to reduced efficiency of the heterologous lactate dehydrogenase. The parental EJ4 strain, which did not express $l d h A$, produced no 
lactic acid, as expected. This experiment served as an initial proof of concept that cow's milk can be converted into other value-added products, which may be useful for industrial-scale dairy farms after further scaled-up tests and optimizations.

Finally, we tested whether our EJ4L strain could efficiently ferment whey from bovine milk to produce lactic acid. Initially, we used whey powder that was not autoclaved, but we observed bacterial contamination in each of our attempted fermentations, indicated by rapid production of acetic acid. To alleviate the contamination issue, we autoclaved the medium to destroy native bacteria before inoculating it with the EJ4L strain. As a result, we were able to conduct our whey fermentations without microbial contaminants. The EJ4L strain could produce $\sim 15.6 \mathrm{~g} / \mathrm{L}$ of lactic acid in $110 \mathrm{~h}$, resulting in a yield of $0.356 \mathrm{~g}$ of lactic acid/g of lactose and a productivity of $0.142 \mathrm{~g} / \mathrm{L} \cdot \mathrm{h}$ (Figure $3)$. We detected no ethanol during the fermentation. The parental EJ4 strain, which did not express $l d h A$, produced no lactic acid, as expected. This experiment provided initial insight into the potential for converting waste whey, such as acid whey, into a value-added product. Because whey is highly nutrient-dense, further experiments may be necessary to test different sources of whey or investigate the importance of the individual components of whey to develop an optimized lactic acid fermentation.

We have demonstrated some initial steps necessary for converting dairy farm waste into value-added products. Lactic acid was the major product, with ethanol being a minor or nondetectable product, from purified lactose (Figure 1), milk (Figure 2), and whey (Figure $3)$. These results were similar to our previous reports of lactic acid being the major product of xylose or cellobiose fermentations by engineered $S$. cerevisiae, which are likely due to multiple factors, including an absence of glucose signaling and the differing enzyme kinetics of pyruvate decarboxylase/alcohol dehydrogenase and lactate dehydrogenase (Turner et al., 2016). Still, several barriers must be crossed before engineered yeast can be used for industrial-scale production of value-added products such as lactic acid from lactose, waste milk, or acid whey.

First, the engineered strain would need to have no antibiotic resistance. Large-scale production of fuels and chemicals using engineered microbes with inherent antibiotic resistance is generally not supported by the United States Food and Drug Administration due to the risk for spreading antibiotic resistance (Bell et al., 2014). In this proof-of-concept study, our engineered EJ4L yeast strain possessed G418 (geneticin) resistance via the integrated pITy3-ldhA-G418 cassette. However, by using auxotrophic markers or the modern CRISPR/
Cas9 (DiCarlo et al., 2013; Zhang et al., 2014) engineering protocol, even in an industrial polyploid yeast strain, it is possible to develop an $l d h A$-expressing yeast strain without the need for an antibiotic marker.

Second, lactic acid productivity $(\mathrm{g} / \mathrm{L} \cdot \mathrm{h})$ would need to be increased to produce an economically viable fermentation process. This could be achieved by improving the lactose fermentation pathway (CDT-1 and GH1-1) through increased copy numbers of the relevant genes. Perhaps more simply, the fermentation rate could be increased by starting with a significantly higher initial yeast cell density, 10- or 100-fold higher than that used in this study. By improving the lactose fermentation pathway and increasing the initial cell density, greater lactic acid productivity could be achieved.

Finally, a rapid and cost-effective purification process is needed to separate spent whey medium and yeast cells from the produced lactic acid. At least 2 separation steps would be needed, including filtration to remove solids and then isolation of lactic acid from the liquid medium. Many purification methods already exist and are sometimes applied to industrial-scale lactic acid fermentation, but they may need to be modified specifically to purify lactic acid from whey medium (Kim and Moon, 2001; Thang et al., 2005).

Introduction of a heterologous lactose fermentation pathway consisting of a lactose transporter (CDT-1) and an intracellular $\beta$-galactosidase (GH1-1) and a heterologous lactic acid production pathway consisting of lactate dehydrogenase into a laboratory $S$. cerevisiae yeast allowed for the production of lactic acid from lactose, cow's milk, or whey, with minimal to no ethanol accumulation. A yield of $0.358 \mathrm{~g}$ of lactic acid/g of lactose from YP medium containing $\sim 80 \mathrm{~g} / \mathrm{L}$ whey was achieved with no detectable ethanol production, suggesting that homolactic acid production from lactose can be achieved in engineered $S$. cerevisiae, even without deletion of the native ethanol pathway.

\section{ACKNOWLEDGMENTS}

TLT acknowledges that this project was supported by the Agriculture and Food Research Initiative Competitive Grant No. 2015-67011-22806 from the United States Department of Agriculture National Institute of Food and Agriculture (Washington, DC). YSJ and JJL are affiliated with the Energy Biosciences Institute (University of Illinois at Urbana-Champaign).

\section{REFERENCES}

Arif, K., M. Islam, A. Maroof, K. Shazia, K. Umber, B. Deepa, M. Owais, E. Cerdán, I. González-Siso and M. Becerra. 2008. Applications of whey in biotechnology. Pages 1-33 in Advances in 
Cheese Whey Utilization. Transworld Research Network, Trivandrum, India.

Bell, B. G., F. Schellevis, E. Stobberingh, H. Goossens, and M. Pringle. 2014. A systematic review and meta-analysis of the effects of antibiotic consumption on antibiotic resistance. BMC Infect. Dis. 14:13. https://doi.org/10.1186/1471-2334-14-13.

Breeuwer, P., and T. Abee. 2000. Assessment of the intracellular pH of immobilized and continuously perfused yeast cells employing fluorescence ratio imaging analysis. J. Microbiol. Methods 39:253-264. https://doi.org/10.1016/S0167-7012(99)00124-4.

DiCarlo, J. E., J. E. Norville, P. Mali, X. Rios, J. Aach, and G. M. Church. 2013. Genome engineering in Saccharomyces cerevisiae using CRISPR-cas systems. Nucleic Acids Res. 41:4336-4343. https://doi.org/10.1093/nar/gkt135.

Elliott, J. 2013. Whey too much: Greek yogurt's dark side. Modern Farmer. Accessed Jul. 25, 2016. http://modernfarmer. com/2013/05/whey-too-much-greek-yogurts-dark-side/.

Galazka, J. M., C. Tian, W. T. Beeson, B. Martinez, N. L. Glass, and J. H. Cate. 2010. Cellodextrin transport in yeast for improved biofuel production. Science 330:84-86. https://doi.org/10.1126/ science. 1192838

González-Siso, M. I., A. Touriño, Á. Vizoso, Á. Pereira-Rodríguez, E. Rodríguez-Belmonte, M. Becerra, and M. E. Cerdán. 2015. Improved bioethanol production in an engineered Kluyveromyces lactis strain shifted from respiratory to fermentative metabolism by deletion of NDI1. Microb. Biotechnol. 8:319-330. https://doi. org $/ 10.1111 / 1751-7915.12160$.

Kim, Y. H., and S. Moon. 2001. Lactic acid recovery from fermentation broth using one-stage electrodialysis. J. Chem. Technol. Biotechnol. 76:169-178. https://doi.org/10.1002/jctb.368.

Laluce, C., A. Schenberg, J. Gallardo, L. Coradello, and S. PombeiroSponchiado. 2012. Advances and developments in strategies to improve strains of Saccharomyces cerevisiae and processes to obtain the lignocellulosic ethanol-A review. Appl. Biochem. Biotechnol. 166:1908-1926. https://doi.org/10.1007/s12010-012-9619-6.

Liu, J. J., G. C. Zhang, E. J. Oh, P. Pathanibul, T. L. Turner, and Y. S. Jin. 2016. Lactose fermentation by engineered Saccharomyces cerevisiae capable of fermenting cellobiose. J. Biotechnol. 234:99 104. https://doi.org/10.1016/j.jbiotec.2016.07.018.

Miller, G. D., J. K. Jarvis, and L. D. McBean. 2006. Handbook of Dairy Foods and Nutrition. CRC Press, Boca Raton, FL.

Oh, E. J., J. M. Skerker, S. R. Kim, N. Wei, T. L. Turner, M. J. Maurer, A. P. Arkin, and Y. S. Jin. 2016. Gene amplification on demand accelerates cellobiose utilization in engineered Saccharomyces cerevisiae. Appl. Environ. Microbiol. 82:3631-3639. https:// doi.org/10.1128/AEM.00410-16.

Parekh, R. N., M. R. Shaw, and K. D. Wittrup. 1996. An integrating vector for tunable, high copy, stable integration into the dispersed Ty $\delta$ sites of Saccharomyces cerevisiae. Biotechnol. Prog. 12:16-21. https://doi.org/10.1021/bp9500627.

Porro, D., L. Brambilla, B. M. Ranzi, E. Martegani, and L. Alberghina. 1995. Development of metabolically engineered Saccharomyces cerevisiae cells for the production of lactic acid. Biotechnol. Prog. 11:294-298. https://doi.org/10.1021/bp00033a009.

Siso, M. G. 1996. The biotechnological utilization of cheese whey: A review. Bioresour. Technol. 57:1-11. https://doi.org/10.1016/09608524(96)00036-3.

Skory, C. D. 2003. Lactic acid production by Saccharomyces cerevisiae expressing a Rhizopus oryzae lactate dehydrogenase gene. J. Ind. Microbiol. Biotechnol. 30:22-27. https://doi.org/10.1007/s10295002-0004-2.

Stabel, J. R. 2001. On-farm batch pasteurization destroys Mycobacterium paratuberculosis in waste milk. J. Dairy Sci. 84:524-527. https://doi.org/10.3168/jds.S0022-0302(01)74503-1.

Thang, V. H., W. Koschuh, K. D. Kulbe, and S. Novalin. 2005. Detailed investigation of an electrodialytic process during the separation of lactic acid from a complex mixture. J. Membr. Sci. 249:173-182. https://doi.org/10.1016/j.memsci.2004.08.033.

Turner, T. L., G. Zhang, E. J. Oh, V. Subramaniam, A. Adiputra, V. Subramaniam, C. D. Skory, J. Y. Jang, B. J. Yu, and I. Park. 2016. Lactic acid production from cellobiose and xylose by engineered Saccharomyces cerevisiae. Biotechnol. Bioeng. 113:1075-1083. https://doi.org/10.1002/bit.25875.

Wei, N., E. J. Oh, G. Million, J. H. Cate, and Y. Jin. 2015. Simultaneous utilization of cellobiose, xylose, and acetic acid from lignocellulosic biomass for biofuel production by an engineered yeast platform. ACS Synth. Biol. 4:707-713. https://doi.org/10.1021/ sb500364q.

Zhang, G. C., I. I. Kong, H. Kim, J. J. Liu, J. H. Cate, and Y. S. Jin. 2014. Construction of a quadruple auxotrophic mutant of an industrial polyploid Saccharomyces cerevisiae strain by using RNAguided Cas9 nuclease. Appl. Environ. Microbiol. 80:7694-7701. https://doi.org/10.1128/AEM.02310-14. 